\title{
Remote Patient Management in Peritoneal Dialysis: An Answer to an Unmet Clinical Need
}

\author{
Oommen John ${ }^{\mathrm{a}, \mathrm{b}} \cdot$ Vivekanand Jha ${ }^{\mathrm{a}-\mathrm{c}}$
}

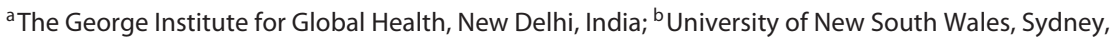
NSW, Australia; ' University of Oxford, Oxford, UK

\begin{abstract}
The burden of chronic kidney disease is increasing globally. Novel methods for the management of end-stage kidney disease at home have been available for several years, however uptake of home peritoneal dialysis (PD) has been suboptimal for a variety of reasons. Nonadherence is an important factor that determines the outcomes of PD; patients on home dialysis are subject to feeling isolated and are anxious to lack of routine clinical oversight. When patients feel disconnected from their health care professionals, their compliance to medical advice drops and their confidence in self-care comes down. Remote patient management (RPM) has the potential to improve outcomes in PD through telehealth platforms that facilitate virtual clinical presence, enable patient-generated clinical documentation and feedback mechanism, and promote self-monitoring. Bi-directional communications between patients and clinicians provide an enabling environment for autonomy while being clinically monitored through a co-presence, resulting in collaborative care that could alleviate the anxiety of the patients about not being under the direct care of a physician. RPM enables the clinicians to closely monitor and detect early issues, provide feedback in realtime, and initiate early interventions such as prescription modifications and contextual clinical decision support. As the computational capabilities improve and clinical data are collated, machine learning and artificial intelligence algorithms would help detect patterns and predict impending complications such as fluid overload, heart failure or peritonitis, thereby allowing early detection and interventions to avoid hospitalizations. The technical framework and essential features for a RPM system in PD is outlined in this chapter.
\end{abstract}




\section{Introduction}

Societies worldwide are confronting the problem of managing the ever-growing population of patients with end-stage kidney disease (ESKD), driven by population growth, aging, obesity, diabetes, and hypertension. The Global Burden of Disease estimates that chronic kidney disease will become the 5 th leading cause of death by 2040, up from the current 16th position [1]. Renal replacement therapy (RRT), in the form of hemodialysis (HD), peritoneal dialysis (PD) or renal transplantation can add many meaningful years to the lives of patients with ESKD. In suitable candidates, kidney transplant is the preferred option, but only a minority can receive this treatment, largely because of organ shortage [2]. A systematic review [3] estimated the number of patients on RRT worldwide in 2010 at 2.62 million, with 2.05 million being on dialysis. A majority of these patients received in-center HD. The same review estimated that at least 2.3 million people died that year annually because of lack of access to dialysis, mostly in low and low-middle income countries. The authors estimated that the number of people undergoing RRT will more than double to 5.4 million by 2030 , mostly in low-middle income countries. However, the number of people without access to RRT will remain substantial. This is not a surprise because chronic dialysis is one of the most expensive therapies for individuals and governments. These data show a pressing need to develop innovative cost-effective approaches so that RRT can be made available to most of the people who can benefit from it.

There is plenty of data $[4,5]$ that suggest that compared to in-center HD, home dialysis - either home hemo or PD - can provide equivalent or superior clinical outcomes and quality of life at a lower cost to the healthcare system. Even though most patients are equally suitable for HD or PD [6], a number of patients, physicians, and healthcare system related factors conspire to make incenter HD the default option [7]. However, healthcare policymakers in many countries have realized the need to address this imbalance and are adopting strategies to promote home therapies, a policy review identified Hong Kong, Thailand, and Australia as having PD-first policies [8]. Since PD is less manpower- and resource-intensive, countries that are implementing dialysis as part of universal health coverage tend to favor PD as the preferred option.

In theory, the flexibility of schedule, convenience of performing dialysis at home, preservation of lifestyle should make PD the more attractive option everywhere, especially for the younger patients with fewer comorbidities and busy lifestyle [9]. Other compelling reasons for adopting PD is that it can be administered in remote locations without the need of physical infrastructure. However, according to the Global Kidney Health Atlas of the International Society of Nephrology [10], while HD is available in $95 \%$ of the 125 countries that were 
surveyed, citizens of only $76 \%$ of countries can access PD. Further, even where it is available, only one-third and just about $12 \%$ of ESKD patients beginning maintenance dialysis are presented with $\mathrm{PD}$ and Home HD, respectively, as treatment option [11]. Moreover, 25-40\% of patients would choose a home dialysis modality if the option was presented to them [12].

Patient involvement in influencing health decisions is becoming a central component of healthcare provision around the world. Research has suggested that being given a meaningful choice enhances patient autonomy and self-sufficiency [13]. A meta-analysis on decision aids (educational material focusing on options and outcomes for the purpose of preparing people for decision making) showed that aids compared to usual care helped people feel more informed about their options, more comfortable about their choices, and provided a clearer picture regarding personal values that affect their choices [13].

Provision of information has been clearly associated with an increased uptake of PD. Given the right quantity and quality of information, more patients will opt for PD. Provision of adequate and good quality information in a timely manner [14] should be an integral component of renal care services delivery. However, studies have shown that the written information provided by most renal care services are difficult to understand [15], and contemporary digital tools and mobile platforms are not utilized for health information dissemination and clinician to patient communications, indicating a large scope for improvement.

Studies indicate that PD is more likely to be undertaken by people who are married, living with another person, and are employed or studying. This suggests that patients who live alone may not choose PD purely due to lack of support [16]. Social barriers to self-care PD could be overcome by the provision of home care assistance in the form of assisted PD or remote patient management (RPM).

\section{Rationale for RPM}

Home-based self-care modalities including PD are not without drawbacks, both real and imaginary. After patients and their caregivers have been trained to deliver these complex treatments at home, there is a risk that they might feel abandoned by the healthcare system due to the lack of real-time oversight by trained health professionals, which might increase their anxiety levels [17]. In a survey of patients with recently diagnosed ESKD who had chosen HD over PD, lack of confidence in patient's ability to perform this therapy at home without supervision or feedback from a healthcare provider emerged as an important reason for 
the choice (Jha V., unpublished data). The lack of real-time oversight could also result in non-adherence to lifestyle and dietary advice, medications or dialysis prescriptions such as skipping or shortening dialysis sessions [18].

The feelings of isolation and difficulty in accessing assistance can lead to lack of confidence and poor decision making, including abandoning a useful and effective treatment modality and increasing the risk of complications [19]. The traditional approach to overcoming these barriers is to make frequent phone calls or home visits and use paper-based log books to record parameters of PD treatments undertaken at home. However, the log of these parameters can only be reviewed during a scheduled in-person visit or after a complication has forced the patient to seek care. The major drawback of this asynchronous model of care review and delivery is that it could be a long time until the patient sees their healthcare professionals, rendering it impossible for them to take early anticipatory corrective actions to reduce the odds of future complications.

Currently available technology frameworks and the increasing use of mobile devices have resulted in the development of tools that can be used as simple selfmonitoring or logging apps. Healthcare establishments and medical professionals are also devoting efforts to building customized computer-based self-monitoring systems [20, 21]. Key features of these self-monitoring systems include summative information of vital signs, interactive dashboards for health parameters and laboratory test results, prescriptions, and electronic reminders that are automated to specified frequency and predefined triggers (Table 1). While electronic logs and reminder systems are likely to improve non-compliance, the isolation and lack of real-time oversight are unlikely to be addressed. In the setting of home-based therapies like PD, the patients might continue to feel isolated and anxious about the status of their health even when their dialysis-related parameters are stable and within expected ranges due to the perception that their treatments are not receiving real-time oversight by trained healthcare professionals compared to those opting for in-center HD who receive treatment under direct supervision. Studies have shown that addressing social isolation and catering to the emotional needs of users is a major challenge to emerging telemonitoring and innovative technologies that deliver care remotely [22].

IT-enabled patient monitoring systems are increasingly being deployed in chronic disease management. RPM uses information and communication technologies to transfer medical information from the patient for the delivery of clinical and educational services with an aim to improve health outcomes and simulate a real-time clinician-patient interaction, as illustrated in Figure 1. Collection of information is being increasingly automated with the help of application programming interfaces and sensors that allow seamless capture and transmission of parameters from multiple sources to the system in real-time. RPM 
Table 1. Components of a remote management system for PD

\begin{tabular}{|c|c|c|c|}
\hline Goals & Parameters & Methods & Enabling technologies \\
\hline \multirow[t]{3}{*}{$\begin{array}{l}\text { Self- } \\
\text { monitoring }\end{array}$} & $\begin{array}{l}\text { Pre- and post- } \\
\text { dialysis weight }\end{array}$ & $\begin{array}{l}\text { Patient facing mobile application } \\
\text { with scheduler function that } \\
\text { automatically triggers weight } \\
\text { recording at each exchange session } \\
\text { and ability to visually display value } \\
\text { against goals }\end{array}$ & $\begin{array}{l}\text { Native mobile application with } \\
\text { inbuilt calendar or event-driven } \\
\text { scheduler; Bluetooth protocols for } \\
\text { acquiring weights from an } \\
\text { automated weighing machine }\end{array}$ \\
\hline & Blood pressure & $\begin{array}{l}\text { Automated blood pressure } \\
\text { monitoring with prompts by the } \\
\text { patient interface application, ability } \\
\text { to display recording graphically in a } \\
\text { time series and against target }\end{array}$ & $\begin{array}{l}\text { Computerized algorithm that } \\
\text { triggers recording of BP through } \\
\text { automated machines and } \\
\text { recordings transmitted through } \\
\text { Bluetooth protocols }\end{array}$ \\
\hline & Blood glucose & $\begin{array}{l}\text { Blood glucose monitoring prompted } \\
\text { by the calendar or event triggered } \\
\text { algorithms. Ability to visually display } \\
\text { recordings against target glycemic } \\
\text { control goals }\end{array}$ & $\begin{array}{l}\text { Point of care glucometers with } \\
\text { wireless capability directly transfers } \\
\text { recordings into the patient } \\
\text { application }\end{array}$ \\
\hline \multirow[t]{4}{*}{$\begin{array}{l}\text { Exchange- } \\
\text { related } \\
\text { parameters }\end{array}$} & $\begin{array}{l}\text { Aseptic } \\
\text { techniques } \\
\text { followed }\end{array}$ & $\begin{array}{l}\text { Periodic evaluation for adherence to } \\
\text { aseptic techniques through a } \\
\text { checklist in the patient interface. } \\
\text { Reinforcement of aseptic technique } \\
\text { training in case of technique failure } \\
\text { noted or complications such as } \\
\text { peritonitis, exit site infections }\end{array}$ & $\begin{array}{l}\text { Rules engine-driven algorithms that } \\
\text { collect data on the checklists, } \\
\text { graphical user interface for ease of } \\
\text { data collection }\end{array}$ \\
\hline & $\begin{array}{l}\text { Adherence to } \\
\text { exchange } \\
\text { schedule }\end{array}$ & $\begin{array}{l}\text { Recording of the actual exchanges } \\
\text { against the prescription through } \\
\text { patient interface }\end{array}$ & $\begin{array}{l}\text { Prescription-driven workflows that } \\
\text { define and capture data, algorithms } \\
\text { to prompt and alert when } \\
\text { deviations from prescriptions are } \\
\text { recorded }\end{array}$ \\
\hline & Dwell time & $\begin{array}{l}\text { Automated stop watch trigger } \\
\text { through voice commands (for } \\
\text { maintaining aspectic conditions) } \\
\text { that enables start and end times of } \\
\text { each PD session }\end{array}$ & $\begin{array}{l}\text { Voice recognition software } \\
\text { capabilities built into the patient } \\
\text { interface application } \\
\text { Local language support essential } \\
\text { for ease of use }\end{array}$ \\
\hline & $\begin{array}{l}\text { PD fluid and exit } \\
\text { site characteristics }\end{array}$ & $\begin{array}{l}\text { Ability to record any change in exit } \\
\text { site or effluent fluid characteristic } \\
\text { through questionnaires and } \\
\text { triggering of an action pathway } \\
\text { when abnormal }\end{array}$ & $\begin{array}{l}\text { In-built questionnaire trigger from } \\
\text { rules engine, ability to activate } \\
\text { device camera to capture a visual } \\
\text { record, Al-based response }\end{array}$ \\
\hline $\begin{array}{l}\text { General } \\
\text { wellbeing }\end{array}$ & $\begin{array}{l}\text { Overall health } \\
\text { status and specific } \\
\text { comorbid } \\
\text { conditions }\end{array}$ & $\begin{array}{l}\text { Adaptive questionnaire, tailored } \\
\text { display of an interactive interface }\end{array}$ & $\begin{array}{l}\text { CDS rules engine allows tailored } \\
\text { generation of questionnaire based } \\
\text { on patient characteristics through a } \\
\text { web service, API over secure layer }\end{array}$ \\
\hline $\begin{array}{l}\text { Emotional } \\
\text { wellbeing } \\
\text { and quality } \\
\text { of life }\end{array}$ & $\begin{array}{l}\text { Emotional } \\
\text { wellbeing, quality } \\
\text { of life and mental } \\
\text { health }\end{array}$ & $\begin{array}{l}\text { Emotional wellbeing being captured } \\
\text { through a graphical interface. } \\
\text { Deviations triggering predefined } \\
\text { pathways to determine mental } \\
\text { health status through administration } \\
\text { of standardized tools }\end{array}$ & $\begin{array}{l}\text { Interactive graphical interface on } \\
\text { the patient application } \\
\text { Advanced technologies such as } \\
\text { facial recognition coupled with } \\
\text { machine learning algorithms to } \\
\text { infer mood status through pattern } \\
\text { recognition }\end{array}$ \\
\hline
\end{tabular}

$\mathrm{PD}$, peritoneal dialysis; CDS, clinical decision support. 


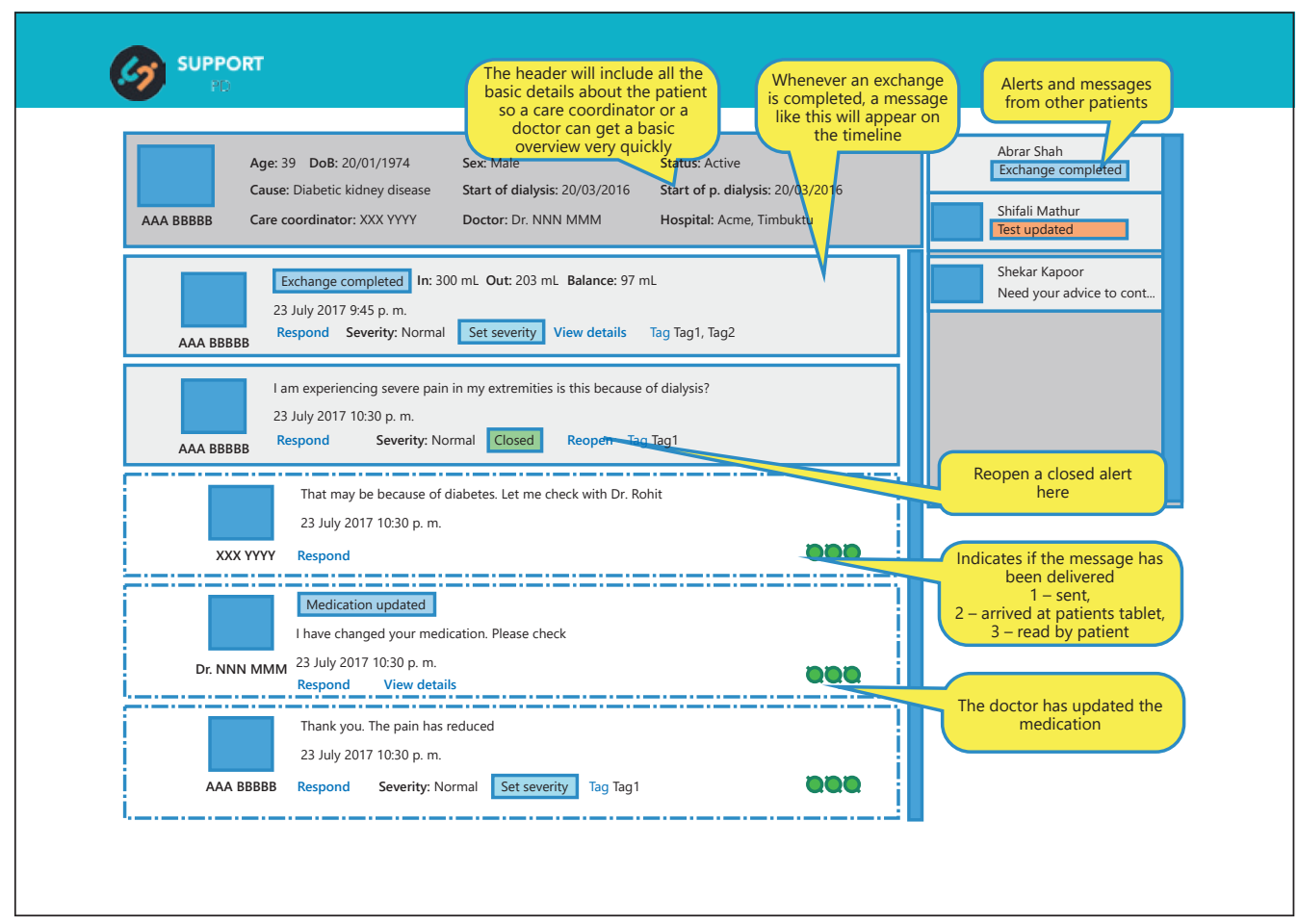

Fig. 1. Interactive communication between patient and physician. SUPPORT PD, Seamless User Centered Proactive Provision of Care in PD.

provides a framework for monitoring patients at home by digital wireless technology and facilitates the extension of the continuum of care into the patients' home setting through interactive technological interfaces. These technologies have the potential to improve clinical outcomes through improved patient engagement, and earlier recognition and correction of impending complications [23].

RPM systems have been used to monitor a number of chronic conditions, including heart failure [24], diabetes [25], mental illnesses [26], asthma [27], and obesity [28]. Meta-analyses have shown the benefits of structured telephone support, RPM, and the use of implantable electronic devices in the care of patients with cardiac diseases [29]. Telehealth programs with the ability to change medications remotely have been shown to lower glycosylated hemoglobin in diabetics as compared to standard of care [30].

RPM provides an opportunity to increase the uptake and technique survival of the home dialysis modalities by improving patient satisfaction and outcomes, and lead to cost savings [31]. The evidence-base for the utility of RPM in home dialysis is still emerging. Small observational studies suggest the technical feasi- 
bility and numerous potential benefits of RPM [32,33]. However in general, nephrology has been slow to accept telehealth technology, in part due to regulations surrounding telehealth implementation, including information security considerations and reimbursement policies [31].

\section{Features of a RPM System for PD}

\section{Augmented Communication between Patients and Healthcare Delivery} Systems Resulting in Efficient and Sustainable Self-Care

PD patients spend a significant amount of time in self-care tasks. Enabling robust bidirectional communication with healthcare providers has the potential to not only reduce the burden of these activities but also to improve patient satisfaction and quality of life [31]. The communication features could be used for regular and patient-initiated on-demand teleconsultations, thereby reducing the time spent in travel and waiting rooms, save on travel costs, and enhance user experience for the patient. This is important because PD patients on an average need to travel greater distances to get to their care provider than patients traveling to in-center HD units [34]. Communication systems with imaging transmission or video-conferencing capabilities may allow early identification of common medical problems such as peritonitis, exit site infections, and volume overload [23]. Interactive questionnaires with graphical user friendly interface such as a tablet application about medical issues and quality of life, could facilitate continuous interaction between patients and provider. Response to simple questions like shortness of breath, swelling, appetite, and exit site and PD fluid appearance can help identify patients who require attention and possible intervention. Advantage could be taken of the built-in voice and image functions of these devices to collect non-text information. Such actions could be automated through built-in algorithms that trigger the most appropriate response - such as a change in the PD prescription, an action from the patient, an in-person visit from a nurse or patient being called to the health facility. Seamless User Centered Proactive Provision of Care in PD is one such system that has been developed, as illustrated in Figure 2.

\section{User-Generated Clinical Data and Non-Invasive Monitoring of Clinical}

Parameters through Wearable Sensors and IoT Devices Resulting in Real-Time Clinical Documentation

PD patients spend time collecting and recording detailed data pertaining to their dialysis treatments, generating paper-based records. Enabling technologies (e.g., Bluetooth or wireless) that already exist can eliminate the need to manually re- 


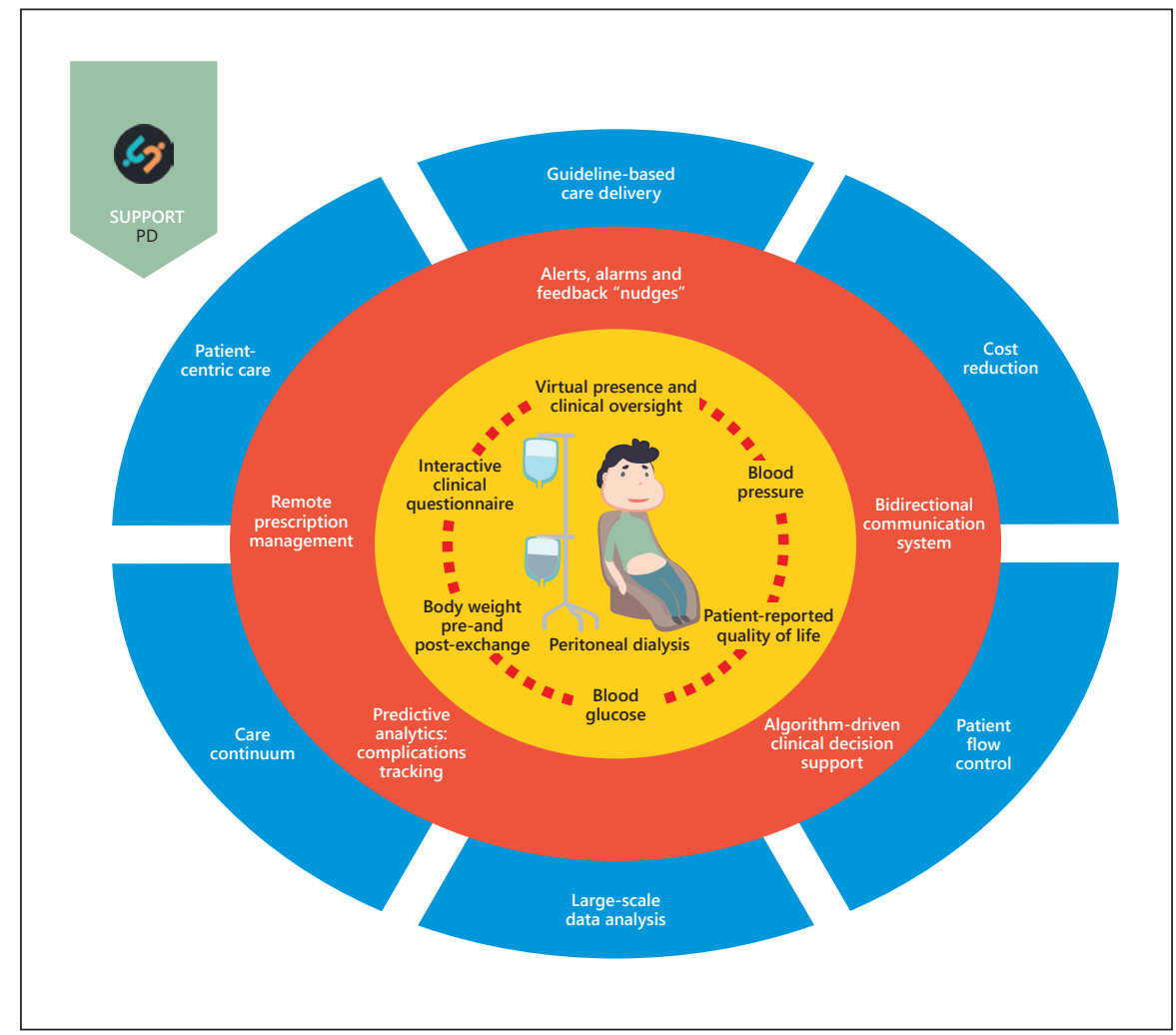

Fig. 2. A remote patient management ecosystem. SUPPORT PD, Seamless User Centered Proactive Provision of Care in PD.

cord data pertaining to weight, vitals, and dialysis treatment parameters. Wearable sensors and Internet of Things based physiological monitoring devices can substantially meet these goals. In fact, this offers the opportunity to monitor more parameters than are currently practiced, such as using an oximeter to detect nocturnal hypoxemia, which has been associated with cardiovascular complications in dialysis patients [35].

These approaches will reduce the time taken for recording data, increase data accuracy and the frequency with which that information is collected, transmitted and reviewed by care providers leading to enhanced oversight, and improve both physician and patient comfort levels, allaying fears of self mismanagement.

Collected data should be able to integrate into existing electronic health records so as not to lead to needless duplication of data entry. The ability of clinicians and their home dialysis patients to have visibility and track weights, ultrafiltration (UF), and blood pressures would contribute to better volume control [31] and trigger intervention as needed. The ability to monitor treatment adher- 
ence is important with respect to patient outcomes, as it has been shown to be an indicator for the risk of developing peritonitis, hospitalization, hospital days, technique failure, and death [36]. Futuristic RPM care models that use data analytics to convert information to a more usable form and provide algorithms to flag concerning values or trends are needed as the burden of ESKD is increasing.

\section{Automated Clinical Decision Support System (CDSS)-Driven System That} Will Respond Immediately to Patient Input and Provide Advice Consistent with Guidelines on the Management of Their Condition

As soon as patient or device generated data become available, automated monitoring through the built-in applied intervention algorithms could provide interactive feedback to the patients and help them follow recommended practices for improved outcomes, such as those related to volume management, blood pressure, and glycemic control. Adherence to prescription, cycler alarms, UF values, vital signs, and weights on a more frequent basis will prevent unnecessary hospitalizations. RPMs with automated response systems are able to "nudge" patients into practices consistent with current guidelines. An important feature of such a system would be the ability to seamlessly update the algorithm at the back end when there is a change in guideline. As large sets of data become available, machine learning techniques could be applied to identify specific patterns that predict complications and trigger interventions to prevent complications.

\section{Patient Education and Support System}

A key to success of home-based dialysis, particularly PD, is patient education and training. The value of properly designed training protocol and retraining has been shown in multiple studies. RMP provides opportunities for e-learning or nurse-to-patient teaching using education modules that are standardized to improve patient knowledge regarding self-care. Education could also be done via 2-way communications and allow clinicians to directly visualize PD exchanges and provide real-time feedback to improve PD technique [31].

\section{Electronic Prescription Management Facilitating Personalized Therapy in Real-Time}

Management of medication and PD prescription would be feasible with RPM platforms. For example, in a patient who shows serial weight gain, an algorithm could provide automated response based on pre-set thresholds and at the same time alert the physician to review the dialysis prescription and modify the frequency or type of dialysate to augment UF, review salt and fluid intake or adjust diuretic doses. A similar feature could be used for blood pressure and glycemia management. 
6. Virtual Presence or Copresence by Which a Sense of Connection with Another in a Mediated or Online Environment Is Real, Immediate, or Present

When patients feel disconnected from their healthcare professionals, their compliance to medical advice drops and their confidence of self-care comes down [17]. Recent meta-analysis have attributed loneliness to be a risk factor for mortality [37]. Patients undertaking long-term home dialysis are subject to feelings of isolation and anxiety due to the absence of physical contact with their healthcare professionals and lack of feedback in regards to their dialysis treatment. Technology enablement in these patients to feel the "presence" of the healthcare professionals remotely while on dialysis at home for better compliance with the dialysis regime and to feel connected with healthcare professionals could result in "co-presence" [38]. A virtual presence or co-presence can be achieved through a multimodal interactive engagement between the clinician, healthcare delivery system, and the patient through avatars or role-based characters who provide feedback through communication technologies, augmented through audio/video interactions at specified frequencies or during any health events.

\section{Specific Features for RPM in PD}

\section{Self-Monitoring Applications}

These applications allow the patients to record their PD exchange details such as body weight, blood pressures, exchange volumes, and session times into the electronic interface manually or automatically through wireless technologies and get instant alerts on abnormal parameters or any deviation from their dialysis prescriptions.

\section{Communication System}

The system should allow the patient to communicate with the doctor, dialysis nurse or other authorized healthcare delivery personnel, through voice call, text message or email.

\section{Alerts}

Automated alerts related to deviation from a pre-set parameter, such as the characteristic of drained fluid being different from transparent or UF not being as expected, weight gain/shortness of breath suggesting fluid overload, changes in mood indicating depression. 


\section{Clinical Decision Support System}

As an integral part of the RPM, the clinical decision support would be a rulesbased engine or advanced computational system that can leverage machine learning, artificial intelligence for pattern recognition, pattern-based prediction to identify those at risk of complications, hospitalization or death. The predictive analytic capabilities along with built-in guideline-based clinical decision support could promptly suggest appropriate evidence-based interventions.

\section{Measuring Care Quality and Tracking Outcomes}

Inbuilt data analytics would help track the quality of PD care and clinically relevant outcomes (e.g., peritonitis, fluid overload, exit site infections) and identify associated factors which would encourage a culture of quality monitoring for PD in the home setting.

\section{Technology Framework for RPM in PD}

RPM equipment for PD should include the capacity for safe bidirectional communication between patient and provider. For the patient interface tablet computers or mobile phone devices capable of acquiring images and hosting native mobile application with security, remote data protection features would be ideal. The patient interface should be easy to use and have local language capabilities as well as access to education resources such as training manuals/videos, lifestyle and dietary guidelines, step-by step procedures and a help menu. It should also have an assessment tool that would test the patient's proficiency.

The equipment should have the ability to connect even in locations that may not have access to cable-based Internet providers and should be capable of working offline and synchronize data when internet is available. Methods including either satellite internet or mobile technologies should be incorporated.

Regular communication might include some of the following items: text messages, video (based on internet bandwidth availability), synchronous and asynchronous voice messaging, high-resolution pictures, and the ability of the patient and/or provider to visualize key data in a dashboard format.

Ideally, data collection should be automated (i.e., Bluetooth capable) to prevent patient fatigue with manual entry of data and to ensure data accuracy. RPM equipment should have the ability to connect to the internet in a variety of locations, as remote management may be most useful where access to broadband internet is least available. Equipment should be portable so that it can be used during travel. Equipment and software should be customizable to ensure integration into any clinic's workflow or electronic medical record [31] while ensuring compliance to health data security standards through encrypted data transfer over secure protocols. For seamless interchange of electronic health records 
between RPM and healthcare delivery systems, there is a need to ensure syntactic and semantic interoperability through applicable standards, such as HL 7, SNOMED CT, LOINC, RxNORM etc.

\section{Overarching System for a Hybrid Care Delivery in PD}

Irrespective of the modalities used in remote monitoring, the actual delivery of care for patients on dialysis necessitates periodic face-to-face clinical interaction. Information and tele communication tools are facilitatory tools designed to overcome the misdistribution of the demand versus supply in health systems, in particular the availability of care providers with appropriate clinical skills during the time of need.

Healthcare provision in chronic diseases is becoming extremely complex and beyond the scope of any single care provider. Increasing longevity and chronic disease management in a multimorbidity scenario needs a multidisciplinary and team approach which creates a challenge for domiciliary care. When coordinated care and continuum of care is not followed, quality suffers and impacts outcomes. The team-based approach with multiple providers jointly managing the chronic and multimorbidities towards improving care quality has created a need for seamless communication across elements of healthcare delivery. The model of care components should be clearly identified and defined first, rather than jumping to a solution and getting a piece of technology as a hammer looking for a nail. Ideally, the model of care should be developed by applying principles of user-centered design and design thinking approach; subsequently, the technology solution or system solution that would best fit the RPM care model should be developed.

\section{Looking Ahead: RPM in the Age of Machine Learning and Artificial Intelligence}

Remote health management offers a promising alternative to optimally utilize information and communication technologies for remote diagnosis, monitoring, and delivery of care. However, a number of system-level challenges have prevented these transformative telehealth models from being scaled up at national or regional levels to exploit the full potential of delivering healthcare over distance. Increasing reach and affordability of mobile phones, handheld devices and internet, and availability of open-source software have enabled populations around the world, including in emerging economies to leverage these technologies for mobile banking, tele-education, and a plethora of mobile application based e-commerce ecosystems. Increasingly, patients and healthcare providers are using mobile com- 
munication tools and internet-based messaging platforms for enhancing patientclinician interaction and exchange of clinical information. A technological framework for RPM that is standards compliant, secure, and interactive with capabilities for immediate triaging and responses would enable the health systems to respond to the emerging tsunami of non-communicable diseases.

As part of push towards universal health coverage for ESKD, it is imperative that nephrology community adopts these technologies to help improve patient experience, outcome, and reduce the cost of care. As described above, RPM is particularly suited to support expanding ESKD care delivery in the communities using PD. As technologies advance, early recognition of signs and symptoms of forthcoming complications such as fluid overload, heart failure, peritonitis or sepsis through leveraging the emerging computational techniques in machine learning and artificial intelligence could help prevent or postpone hospitalization and avert the related costs. As patients live longer on PD, concerns related to multimorbidity, frailty, fatigue, and falls would require coupling other emerging areas such as robotic assistance and assisted living. Such a convergence of medical and engineering sciences holds immense potential to improve the health of the nations.

\section{References}

1 Foreman KJ, Marquez N, Dolgert A, Fukutaki K, Fullman N, McGaughey M, Pletcher MA, Smith AE, Tang K, Yuan CW, Brown JC, et al: Forecasting life expectancy, years of life lost, and all-cause and cause-specific mortality for 250 causes of death: reference and alternative scenarios for 2016-40 for 195 countries and territories. Lancet 2018;392:2052-2090.

2 de Fijter JW: Kidney allocation: where utility and fairness meet. Nephrol Dial Transplant 2010;6: 1746-1749.

3 Liyanage T, Ninomiya T, Jha V, Neal B, Patrice HM, Okpechi I, Zhao MH, Lv J, Garg AX, Knight J, Rodgers A, et al: Worldwide access to treatment for end-stage kidney disease: a systematic review. Lancet 2015;385:1975-1982.

4 Weinhandl ED, Foley RN, Gilbertson DT, Arneson TJ, Snyder JJ, Collins AJ: Propensity-matched mortality comparison of incident hemodialysis and peritoneal dialysis patients. J Am Soc Nephrol 2010;21:499-506.

5 Vonesh EF, Snyder JJ, Foley RN, et al: Mortality studies comparing peritoneal dialysis and hemodialysis: what do they tell us? Kidney Int Suppl 2006;103:S3-S11.

6 Mendelssohn DC, Mujais SK, Soroka SD, et al: A prospective evaluation of renal replacement therapy modality eligibility. Nephrol Dial Transplant 2009;24:555-561.
7 Nesrallah G, Mendelssohn DC: Modality options for renal replacement therapy: the integrated care concept revisited. Hemodial Int 2006;10:143-151.

8 Liu FX, Gao X, Inglese G, Chuengsaman P, Pecoits-Filho R, Yu A: A global overview of the impact of peritoneal dialysis first or favored policies: an opinion. Perit Dial Int 2015;35:406-420.

9 Stack AG: Determinants of modality selection among incident US dialysis patients: results from a national study. J Am Soc Nephrol 2002;13: 1279-1287.

10 Bello AK, Levin A, Tonelli M, et al: Assessment of global kidney health care status. JAMA 2017;317: 1864-1881.

11 Mehrotra R, Marsh D, Vonesh E, Peters V, Nissenson A: Patient education and access of ESRD patients to renal replacement therapies beyond in-center hemodialysis. Kidney Int 2005;68:378390.

12 Maaroufi A, Fafin C, Mougel S, et al: Patients' preferences regarding choice of end-stage renal disease treatment options. Am J Nephrol 2013;37: 359-369.

13 O'Connor AM, Stacey D, Entwistle V, et al: Decision aids for people facing health treatment or screening decisions (Review). Cochrane Database Syst Rev 2009;3:CD001431. 
14 Morton RL, Tong A, Howard K, et al: The views of patients and carers in treatment decision making for chronic kidney disease: systematic review and thematic synthesis of qualitative studies. BMJ 2010;340:c112.

15 Bekker H, Winterbottom A, Mooney A: Patient information and decision-making processes. $\mathrm{Br} \mathrm{J}$ Ren Med 2009;14:28-30.

16 Chanouzas D, Ng KP, Fallouh B, Baharani J: What influences patient choice of treatment modality at the pre-dialysis stage? Nephrol Dial Transplant 2011;27:1542-1547.

17 Richmond JM, Lindsay RM, Burton HJ, Conley J, Wai L: Psychological and physiological factors predicting the outcome on home hemodialysis. Clin Nephrol 1982;17:109-113.

18 Leggat JE Jr, Orzol SM, Hulbert-Shearon TE, Golper TA, Jones CA, Held PJ, et al: Noncompliance in hemodialysis: predictors and survival analysis. Am J Kidney Dis 1998;32:139-145.

19 Peters A: Safety issues in home dialysis. Nephrol Nurs J 2014;41:89-92.

20 Faurholt-Jepsen M, Munkholm K, Frost M, Bardram JE, Kessing LV: Electronic self-monitoring of mood using IT platforms in adult patients with bipolar disorder: a systematic review of the validity and evidence. BMC Psychiatry 2016;16:7.

21 Or C, Tao D: A 3-month randomized controlled pilot trial of a patient-centered, computer-based self-monitoring system for the care of type 2 diabetes mellitus and hypertension. J Med Syst 2016; 40:81.

22 Milligan C, Roberts C, Mort M: Telecare and older people: who cares where? Soc Sci Med 2011;72: 347-354.

23 Gallar P, Vigil A, Rodriguez I, et al: Two-year experience with telemedicine in the follow-up of patients in home peritoneal dialysis. J Telemed Telecare 2007;13:288-292.

24 Karhula T, Vuorinen AL, Rääpysjärvi K, Pakanen M, Itkonen P, Tepponen M, et al: Telemonitoring and mobile phone-based health coaching among finnish diabetic and heart disease patients: randomized controlled trial. J Med Internet Res 2015;17:e153.

25 Katalenich B, Shi L, Liu S, Shao H, McDuffie R, Carpio G, et al: Evaluation of a remote monitoring system for diabetes control. Clin Ther 2015; 37:1216-1225.

Prof. Vivekanand Jha

The George Institute for Global Health

310-11 Elegance Tower, Jasola District Centre

New Delhi 110025 (India)

E-Mail vjha@georgeinstitute.org.in
26 Naslund JA, Marsch LA, McHugo GJ, Bartels SJ: Emerging mHealth and eHealth interventions for serious mental illness: a review of the literature. J Ment Health 2015;24:321-332.

27 Finkelstein J, Cabrera MR, Hripcsak G: Internetbased home asthma telemonitoring: can patients handle the technology? Chest 2000;117:148-155.

28 Luley C, Blaik A, Reschke K, Klose S, Westphal S: Weight loss in obese patients with type 2 diabetes: effects of telemonitoring plus a diet combination - the active body control (ABC) program. Diabetes Res Clin Pract 2011;91:286-292.

29 Inglis SC, Clark RA, McAlister FA, et al: Which components of heart failure programmes are effective? A systematic review and meta-analysis of the outcomes of structured telephone support or telemonitoring as the primary component of chronic heart failure management in 8323 patients: abridged cochrane review. Eur J Heart Fail 2011;13:1028-1040.

30 Faruque LI, Wiebe N, Ehteshami-Afshar A, et al: Effect of telemedicine on glycated hemoglobin in diabetes: a systematic review and meta-analysis of randomized trials. CMAJ 2017;189:E341-E364.

31 Wallace EL, Rosner MH, Alscher MD, Schmitt CP, Jain A, Tentori F, Firanek C, Rheuban KS, Florez-Arango J, Jha V, Foo M: Remote patient management for home dialysis patients. Kidney Int Rep 2017;2:1009-1017.

32 Nayak A, Karopadi A, Antony S, et al: Use of a peritoneal dialysis remote monitoring system in India. Perit Dial Int 2012;32:200-204.

33 Ong SW, Jassal SV, Miller JA, et al: Integrating a smartphone-based self-management system into usual care of advanced CKD. Clin J Am Soc Nephrol 2016;11:1054-1062.

34 Prakash S, Coffin R, Schold J, Lewis SA, et al: Travel distance and home dialysis rates in the United States. Perit Dial Int 2014;34:24-32.

35 Zoccali C, Mallamaci F, Tripepi G: Nocturnal hypoxemia predicts incident cardiovascular complications in dialysis patients. J Am Soc Nephrol 2002;13:729-733.

36 Bernardini J, Nagy M, Piraino B: Pattern of noncompliance with dialysis exchanges in peritoneal dialysis patients. Am J Kidney Dis 2000;35:11041110.

37 Rico-Uribe LA, Caballero FF, Martín-María N, Cabello M, Ayuso-Mateos JL, Miret M: Association of loneliness with all-cause mortality: a meta-analysis. PloS One 2018;13:e0190033.

38 Liu N, Kim J, Jung Y, Arisy A, Nicdao MA, Mikaheal M, Baldacchino T, Khadra M, Sud K: Remote monitoring systems for chronic patients on home hemodialysis: field test of a copresence-enhanced design. JMIR Hum Factors 2017;4:e21. 